\title{
Lipidomics of the sea sponge Amphimedon queenslandica and implication for biomarker geochemistry
}

\author{
D. A. Gold \\ \begin{tabular}{l|l} 
J. O. Krömer & R. E. Summons \\
& 1
\end{tabular}
}

${ }^{1}$ Department of Earth, Atmospheric and Planetary Science, Massachusetts Institute of Technology, Cambridge, MA, USA

${ }^{2}$ School of Biological Sciences, University of Queensland, Brisbane, QLD, Australia

${ }^{3}$ Department for Solar Materials, Helmholtz Center for Environmental Research GmbH UFZ, Leipzig, Germany

Correspondence

D. A. Gold, Caltech, Pasadena, CA, USA.

Email: dagold@caltech.edu

and

R. E. Summons, MIT, Cambridge, MA, USA.

Email: rsummons@mit.edu

Present address

D. A. Gold, Division of Biology and Biological Engineering, California Institute of Technology, Pasadena, CA, USA

S. O'Reilly, School of Earth

Sciences, University College Dublin, Belfield, Ireland

Funding information

NASA Astrobiology Institute, Grant/ Award Number: NNA13AA90A; Agouron Institute Geobiology Fellowship; Marie Skłodowska-Curie Actions Programme; Irish Research Council, Grant/Award Number: ELEVATEPD/2014/47

\begin{abstract}
Demosponges are a rich natural source of unusual lipids, some of which are of interest as geochemical biomarkers. Although demosponges are animals, they often host dense communities of microbial symbionts, and it is therefore unclear which lipids can be synthesized by the animal de novo, and which require input from the microbial community. To address this uncertainty, we analyzed the lipids of Amphimdeon queenslandica, the only demosponge with a published genome. We correlated the genetic and lipid repertoires of A. queenslandica to identify which biomarkers could potentially be synthesized and/or modified by the sponge. The fatty acid profile of A. queenslandica is dominated by an unusual $\Delta^{5,9}$ fatty acid (cis-5,9-hexacosadienoic acid)similar to what has been found in other members of the Amphimdeon genus-while the sterol profile is dominated by $\mathrm{C}_{27}-\mathrm{C}_{29}$ derivatives of cholesterol. Based on our analysis of the A. queenslandica genome, we predict that this sponge can synthesize sterols de novo, but it lacks critical genes necessary to synthesize basic saturated and unsaturated fatty acids. However, it does appear to have the genes necessary to modify simpler products into a more complex "algal-like" assemblage of unsaturated fatty acids. Ultimately, our results provide additional support for the poriferan affinity of 24-isopropylcholestanes in Neoproterozoic-age rocks (the "sponge biomarker" hypothesis) and suggest that some algal proxies in the geochemical record could also have animal contributions.
\end{abstract}

\section{1 | INTRODUCTION}

Sea sponges-particularly those in the class Demospongiae-are the greatest natural source of diverse lipid compounds, many of which have been studied for their potential medicinal properties and utility as geochemical biomarkers (Thiel et al., 1999, 2002). Unusual lipids discovered in demosponges include $\mathrm{C}_{23}$ to $\mathrm{C}_{31}$ fatty acids, often with $\Delta^{5,9}$ desaturations-sometimes collectively referred to as demospongic acids (Kornprobst \& Barnathan, 2010)-as well as sterols with atypical side-chain modifications such as 24-isopropylcholesterol (Hofheinz \& Oesterhelt, 1979). However, ongoing skepticism exists in treating these lipids as "sponge biomarkers." Part of this reluctance has to do with the fact that these lipids, while rare in nature, are not taxonomically restricted to demosponges (Giner, Zhao, Boyer, Satchwell, \& Andersen, 2009; Gold, O'Reilly, Luo, Briggs, \& Summons, 2016; Kornprobst \& Barnathan, 2010). But the greater concern is that sea sponges host dense and complex communities of microbes. It is therefore unclear how many of the lipids recovered in demosponges are synthesized by the animal de novo, and which require input from the broader holobiont (a term for the animal and its resident microbes).

In principle, this question could be resolved by comparing the lipids found in demosponges with their repertoire of lipid biosynthesis genes. The demosponge with the best assembled and annotated genome is Amphimedon queenslandica from the Great Barrier Reef, Australia 
(Srivastava et al., 2010). The fatty acid profiles of several sponges from the genus Amphimedon have been analyzed previously and are generally dominated by long-chain 5,9 diunsaturated or 5,9,x triunsaturated fatty acids (Carballeira \& Alicea, 2001; Carballeira, Negrón, \& Reyes, 1992; Carballeira \& Shalabi, 1994; Garson et al., 1993). Unfortunately, genetic data have thrown the taxonomy of Amphimedon and its relatives into flux (until recently, A. queenslandica was known as Reniera sp.), meaning these earlier studies may not be informative for understanding the fatty acid repertoire of A. queenslandica (Redmond et al., 2011). So while Amphimedon is considered a sponge with a conventional lipid repertoire (Lawson, Stoilov, Thompson, \& Djerassi, 1988), recent phylogenetic analyses suggest that some "Amphimedon" species might actually be more closely related to genera that make exotic lipids, such as Petrosia and Haliclona (Redmond et al., 2011). The most recent analysis of fatty acids in definitive specimens of A. queenslandica (Watson, Degnan, Degnan, \& Krömer, 2014) is from a study that broadly quantified protein, lipid, carbohydrate, and nucleic acid content. However, the structure of many fatty acids and sterols was left unresolved, in part because lipids were not derivatized prior to analysis. Given this uncertainty, a detailed assessment of the genes and lipids of $A$. queenslandica will provide critical insight into the fatty acids that can be synthesized by a sponge de novo.

\section{2 | METHODS}

Specimens of A. queenslandica were collected from Shark Bay, Heron Island, Australia $\left(23^{\circ} 26^{\prime} 37.92^{\prime \prime} \mathrm{S}, 151^{\circ} 55^{\prime} 8.81^{\prime \prime} \mathrm{E}\right)$ on $10 / 7 / 2014$ using previously established protocols (Leys et al., 2008). Biomass samples were cut from four individual sponges spaced at least $50 \mathrm{~m}$ apart, and snap-frozen with liquid nitrogen. These samples were stored at $-80^{\circ} \mathrm{C}$ and transported back to The University of Queensland (St Lucia campus) on dry ice. Total lipids were extracted using hexane:isopropanol (Watson, Brennan, et al., 2014; Watson, Degnan, et al, 2014) and stored in sterile glass sample vials under nitrogen gas at $-80^{\circ} \mathrm{C}$. Four total lipid extracts from this original study were sent to MIT on $5 / 17 / 2015$, and subjected to new lipid analyses.

\section{1 | Lipid analyses}

$26 \mu \mathrm{g}$ of 3-methylheneicosane (ULTRA Scientific, North Kingstown, RI) and $5 \alpha$-androstan-3 $\beta$-ol-17-one (epi-androsterone) (SigmaAldrich, St Louis, MO) was added to each total lipid extract as internal standards. Total fatty acids were analyzed after methylation/transesterification of a $10 \%$ aliquot of the total lipid extract using $0.5 \mathrm{M}$ methanolic $\mathrm{HCl}$ (Supleco, Bellefonte, PA). Total sterols were analyzed after mild alkaline methanolysis $(0.5 \mathrm{M} \mathrm{KOH}$ in methanol; $45 \mathrm{~min}$, $50^{\circ} \mathrm{C}$ ) of another $10 \%$ portion of the total lipid extract, followed by silylation with 1:1 (v/v) N,O-bis-trifluoroacetamide (containing 1\% trimethylchlorosilane) and pyridine $\left(2 \mathrm{~h}, 70^{\circ} \mathrm{C}\right.$ ). One microliter (internal standard concentration $\sim 13 \mathrm{ng}_{\mu} \mathrm{L}^{-1}$ ) of the derivatized extract was injected in splitless mode onto an Agilent 7890A gas chromatograph interfaced with an Agilent $5975 \mathrm{C}$ mass selective detector (MSD). The inlet was equipped with a programmable temperature vaporization (PTV) inlet (Gerstel, Linthicum, MD), which was ramped from 70 to $360^{\circ} \mathrm{C}$ at a rate of $720^{\circ} \mathrm{C} \mathrm{min}{ }^{-1}$. Separation was achieved on a J\&W DB-5MS fused silica capillary column (Agilent: $60 \mathrm{~m} \times 0.25 \mathrm{~mm}$ I.D. and film thickness of $0.25 \mu \mathrm{m}$ ). The MSD source and quadrupole were set at 230 and $150^{\circ} \mathrm{C}$, respectively. The column temperature program was as follows: $70^{\circ} \mathrm{C}$ injection and hold for $2 \mathrm{~min}$, ramp at $10^{\circ} \mathrm{C} \mathrm{min}^{-1}$ to $130^{\circ} \mathrm{C}$, followed directly by a ramp at $4^{\circ} \mathrm{C} \mathrm{min}^{-1}$ to $310^{\circ} \mathrm{C}$, and finally an isothermal hold at $310^{\circ} \mathrm{C}$ for $20 \mathrm{~min}$. The MS was operated in electron impact mode with an ionization energy of $70 \mathrm{eV}$ and a mass scan range set from $\mathrm{m} / \mathrm{z} 50$ to 600 . All reported compounds were confirmed using a combination of mass spectral libraries, interpretation of mass fragmentation patterns, compound retention times and by comparison with literature. In the full-scan mode we employed, the on-column detection limits of our instrument are routinely in the high to low picogram range. We used $13 \mathrm{ng}$ on-column injections of 3-methylheneicosane and epi-androsterone as internal standards to monitor reproducibility and performance. From our derivatized sample extracts, we have calculated the LOD $(3 . S / N)$ to be about $0.15 \mathrm{ng}$ and LOQ $(10 . \mathrm{S} / \mathrm{N})$ to be about $0.5 \mathrm{ng}$. The relative abundances of lipids were calculated from peak areas after gas chromatographyflame ionization detection (Agilent 5975C) analysis using the same conditions described above. Double bond locations of monounsaturated fatty acids were confirmed using dimethyl disulfide adduction (Nichols, Guckert, \& White, 1986) by treating a portion of the extracts with dimethyl disulfide and iodine solution ( $6 \% \mathrm{w} / \mathrm{v}$ in diethyl ether) at $50^{\circ} \mathrm{C}$ for $48 \mathrm{~h}$. The detailed results of lipid analysis are provided in Additional File 1.

\section{2 | Genomic analyses}

Data on metabolic gene pathways were collected from the Kyoto Encyclopedia of Genes and Genomes (KEGG PATHWAY) (Kanehisa \& Goto, 2000). This included A. queenslandica-specific data on fatty acid biosynthesis (pathway entry aqu00061), steroid biosynthesis (aqu00100), and biosynthesis of unsaturated fatty acids (aqu01040). Representatives of proteins purportedly missing from $A$. queenslandica were downloaded from KEGG, and compared to the $A$. queenslandica proteome in the National Center for Biotechnology Information (NCBI) refseq database using BLASTp (e-value cutoff 10e-5). Putative A. queenslandica genes missing from KEGG PATHWAY were verified using reciprocal BLAST and PFAM vetting of conserved domains.

To determine the homology of A. queenslandica desaturase and elovl-like proteins, phylogenetic trees were generated. We queried the PFAM protein family database (http://pfam.xfam.org/) for members of the Fatty acid desaturases (Accession: PF00487; ID: FA_desaturase), as well as the GNS1/SUR4 family (Accession: PF01151; ID: ELO), which includes the elovl-like proteins. All peptide sequences from these families were collected for the following organisms: Homo sapiens, Mus musculus, Drosophila melanogaster, Helobdella robusta, Lottia gigantea, Nematostella vectensis, Trichoplax adhaerens, Amphimedon queenslandica, Salpingoeca rosetta, Monosiga brevicollis, Sphaeroforma arctica, Capsaspora owczarzaki, Saccharomyces cerevisiae, Aureococcus 
anophagefferens, and Arabidopsis thaliana. In both datasets, the resulting sequences were vetted for conserved domains using HMMER (v3.1b2) and the Pfam-A database (Eddy, 2008). The conserved domains were aligned using MUSCLE (Edgar, 2004), and edited by hand to remove identicle sequences and sequence fragments. Maximum likelihood trees were generated from the alignments using RaxML (Stamatakis, 2006) with an LG amino acid substitution model, 4 substitution categories, and 100 bootstraps. The resulting trees created several evolutionarily impossible topologies where A. queenslandica proteins were present as both ancestral and daughter sequences, making it difficult to determine the precise homology of these sequences. We therefore optimized the trees using Notung DL (v 2.9; Chen, Durand, \& Farach-Colton, 2000), which rearranges poorly supported branches in a gene/protein tree to produce the most parsimonious result based on the known evolutionary relationships between species. All alignments and trees are provided in Additional File 2.

\section{3 | RESULTS}

\section{1 | Amphimedon queenslandica contains fatty acids and sterols common to demosponges, as well as lipids typically identified with algae and bacteria}

We identified many fatty acids not described in the initial analysis by Watson, Brennan, et al. (2014), including a number of supposed "algal" and "bacterial" biomarkers. The original study identified hexadecanoic (palmitic) acid ( $\mathrm{C}_{16: 0}$ ), an unknown fatty acid (UU4-FA), and octadecanoic acid $\left(\mathrm{C}_{18.0}\right)$ as the most abundant fatty acids in A. queenslandica. Our analyses suggest that UU4-FA is most likely cis5,9-hexacosadienoic acid $\left(\mathrm{C}_{26: 2} \Delta^{5,9}\right)$, a "demospongic" acid that represents $\sim 25 \%$ of all fatty acids (see Table 1 ). Dienoic demospongic acid methyl esters have characteristic ions at $\mathrm{m} / \mathrm{z} 109$ (cleavage between carbon 7 and 8 in addition to loss of 32 Da from methanol), 141 (cleavage between carbon 7 and 8 ) and $[\mathrm{M}-49]^{+}$. Additional "demospongic" acids recovered include cis-5,9-pentacosadienoic $\left(C_{25: 2} \Delta^{5,9}\right)$, cis5,9,14-hexacosatrienoic $\left(C_{26: 3} \Delta^{5,9,17}\right)$, heptacosadienoic $\left(C_{27: 2} \Delta^{5,9}\right)$, and octacosadienoic $\left(\mathrm{C}_{28: 2} \Delta^{5,9}\right)$ acid. On average, these "demospongic" acids make up $\sim 31 \%$ of total fatty acid abundance in A. queenslandica. In addition, we recovered a number of unsaturated fatty acids often considered algal/protist biomarkers, including eicosatetraenoic (arachidonic) $\left(C_{20: 4} \Delta^{5,8,11,14}\right)$, eicosapentaenoic (EPA; $\left.C_{20: 5} \Delta^{5,8,11,14,17}\right)$, docosapentaenoic (DPA; $C_{22: 5} \Delta^{4,7,10,14,16}$ ), and docosahexaenoic (DHA; $C_{22: 6} \Delta^{4,7,10,14,16,19}$ ) acid. These compounds collectively represent $\sim 1 \%$ of all fatty acids. We also recovered multiple branched fatty acids with a putative bacterial origin, including 9-methyl-hexadecanoic (9Me- $\mathrm{C}_{16: 0}$ ), 7-methyl-5-hexadecenoic $\left(7 \mathrm{Me}-\mathrm{C}_{16: 1} \Delta^{5}\right)$, 15-methyl -hexadecanoic ( $\left.\mathrm{iC}_{17: 0}\right), 14-$ methyl-hexadecanoic $\left(\mathrm{aC}_{17: 0}\right)$, 14-methyl -pentadecanoic $\left(\mathrm{iC}_{16: 0}\right)$, 13-methyl-tetradecanoic $\left(\mathrm{iC}_{15: 0}\right)$, and 12-methyl-tetradecanoic $\left(\mathrm{aC}_{15: 0}\right)$ acid. These compounds make up $\sim 7.1 \%$ of all the fatty acids. The fatty acid profiles for A. queenslandica contrast with those reported for A. viridis, A. complanata, and A. compressa, which were found to have $C_{29: 2} \Delta^{5,9,23}$ and $C_{30: 2} \Delta^{5,9,23}$ as major long-chain fatty acids (Carballeira \& Alicea, 2001; Carballeira \&
TABLE 1 Occurrence and average relative abundance ( $n$ specimens $=4$ ) of fatty acids from Amphimedon queenslandica

\begin{tabular}{|c|c|c|}
\hline Fatty acids & & $\begin{array}{l}\text { Average } \pm \\
\text { range }(n=4)\end{array}$ \\
\hline Tetradecanoic acid & C14:0 & $1.0 \pm 0.5$ \\
\hline 13-methyl-tetradecanoic acid & iC15:0 & $1.4 \pm 1.0$ \\
\hline 12-methyl-tetradecanoic acid & aC15:0 & $0.2 \pm 0.2$ \\
\hline Pentadecanoic acid & C15:0 & $0.2 \pm 0.1$ \\
\hline 14-methyl-pentadecanoic acid & iC16 & $3.0 \pm 8.4$ \\
\hline cis-7-Hexadecenoic acid & $\mathrm{C} 16: 1 \Delta^{7}$ & $1.1 \pm 0.1$ \\
\hline cis-9-Hexadecenoic acid & $\mathrm{C} 16: 1 \Delta^{9}$ & $1.6 \pm 0.4$ \\
\hline Hexadecanoic acid & C16:0 & $14.9 \pm 9.3$ \\
\hline 9-methyl-hexadecanoic acid & 9Me-C16:0 & $1.3 \pm 0.6$ \\
\hline 7-methyl-5-hexadecenoic acid & $7 \mathrm{Me}-\mathrm{C} 16: 1^{\Delta 5 *}$ & $0.3 \pm 0.3$ \\
\hline 15-methyl-hexadecanoic acid & iC17:0 & $0.2 \pm 0.1$ \\
\hline 14-methyl-hexadecanoic acid & aC17:0 & $0.6 \pm 0.4$ \\
\hline Heptadecanoic fatty acid & C17:0 & $0.1 \pm<0.1$ \\
\hline 9-octadecenoic acid & $\mathrm{C} 18: 1 \Delta^{9}$ & $2.1 \pm 0.3$ \\
\hline 11-octadecenoic acid & $\mathrm{C} 18: 1 \Delta^{11}$ & $1.0 \pm 0.1$ \\
\hline Octadecanoic acid & C18:0 & $8.9 \pm 4.4$ \\
\hline Nonadecanoic acid & C19:0 & $0.3 \pm 0.2$ \\
\hline $\begin{array}{l}\text { Eicosatetraenoic acid } \\
\text { (Arachidonic) }\end{array}$ & $\mathrm{C} 20: 4 \Delta^{5,8,11,14}$ & $0.4 \pm 0.3$ \\
\hline Eicosapentaenoic acid (EPA) & $\mathrm{C} 20: 5 \Delta^{5,8,11,14,17}$ & $0.1 \pm<0.1$ \\
\hline cis-11-eicosenoic acid & $\mathrm{C} 20: 1 \Delta^{11 c}$ & $0.8 \pm 0.3$ \\
\hline trans-11-eicosenoic acid & $\mathrm{C} 20: 1 \Delta^{11 \mathrm{t}}$ & $0.2 \pm 0.2$ \\
\hline Eicosanoic acid & C2O:0 & $0.2 \pm 0.1$ \\
\hline Docosapentaenoic acid & $\mathrm{C} 22: 5 \Delta^{4,7,10,14,16}$ & $0.3 \pm 0.3$ \\
\hline Docosahexaenoic acid (DHA) & $\mathrm{C} 22: 6 \Delta^{4,7,10,14,16,19}$ & $0.2 \pm 0.1$ \\
\hline Docosanoic acid & $\mathrm{C} 22: 0$ & $7.1 \pm 2.3$ \\
\hline $\begin{array}{l}\text { methyl-branched tricosanoic } \\
\text { acid }\end{array}$ & $\mathrm{brC22}$ & $0.8 \pm 0.2$ \\
\hline Tricosanoic acid & $\mathrm{C} 23: 0$ & $3.4 \pm 1.1$ \\
\hline cis-17-tetracosenoic acid & $\mathrm{C} 24: 1 \Delta^{17}$ & $3.0 \pm 1.8$ \\
\hline Tetracosanoic acid & $\mathrm{C} 24: 0$ & $5.1 \pm 1.3$ \\
\hline 5,9-pentacosadienoic acid & $\mathrm{C} 25: 2 \Delta^{5,9}$ & $1.6 \pm 0.3$ \\
\hline 5,9,14-hexacosatrienoic acid & $\mathrm{C} 26: 3 \Delta^{5,9,17}$ & $0.8 \pm 0.7$ \\
\hline 5,9-hexacosadienoic acid & $\mathrm{C} 26: 2 \Delta^{5,9}$ & $25.3 \pm 5.4$ \\
\hline 15-hexacosenoic acid & $\mathrm{C} 26: 1 \Delta^{15 *}$ & $1.1 \pm 0.2$ \\
\hline 17-hexacosenoic acid & $\mathrm{C} 26: 1 \Delta^{17}$ & $5.9 \pm 1.5$ \\
\hline 19-hexacosenoic acid & $\mathrm{C} 26: 1 \Delta^{19}$ & $1.1 \pm 0.6$ \\
\hline 5,9-heptacosadienoic acid & $C 27: 2 \Delta^{5,9}$ & $2.9 \pm 0.7$ \\
\hline 5,9-octacosadienoic acid & $\mathrm{C} 28: 2 \Delta^{5,9}$ & $0.1 \pm<0.1$ \\
\hline Unknown & & $1.0 \pm 1.2$ \\
\hline
\end{tabular}

Shalabi, 1994; Carballeira et al., 1992). We also failed to detect brominated long-chain fatty acids, which have been reported as major fatty acids for A. terpenensis (Garson, Zimmermann, Battershill, Holden, \& Murphy, 1994; Garson et al., 1993). 
Our analysis of sterols in A. queenslandica also includes newly identified compounds for this organism (Table 2). The sterol profile is dominated by cholesterol $\left(\mathrm{C}_{27} \Delta^{5} ; 36.0 \%\right.$ of total sterols on average), as reported in Watson, Brennan, et al. (2014). We identified $\beta$-sitosterol $\left(\mathrm{C}_{29} \Delta^{5}\right)$ as the second-most abundant sterol $(\sim 26.3 \%$ of total sterols on average), followed by 24-methylcholesta-5,24(28)-dien -3 $\beta$-ol ( $\mathrm{C}_{28} \Delta^{5,24(28)} ; 11.4 \%$ of total sterols). Other identified sterols were brassicasterol $\left(\mathrm{C}_{28} \Delta^{5,22} ; \sim 7.9 \%\right)$, campesterol $\left(\mathrm{C}_{28} \Delta^{5}\right)$, stigmasterol $\left(\mathrm{C}_{29} \Delta^{5,22}\right)$, fucosterol $\left(\mathrm{C}_{29} \Delta^{5,24(28)}\right)$, and 22-cholest-5,22-dien-3 $\beta$-ol $\left(C_{26} \Delta^{5,22}\right)$. The $C_{28}-C_{29}$ sterols identified here are typically considered biomarkers of plants and algae as opposed to animals like A. queenslandica (Volkman et al., 1998). No $\mathrm{C}_{30}$ sterols were identified in this study, although we cannot discount the possibility of trace amounts being present, but falling below instrumental detection limits (Love et al., 2009).

\section{2 | Genome analysis suggests that "demospongic" and algal biomarkers-but not bacterial biomarkers- can be synthesized by Amphimedon queenslandica}

To determine whether A. queenslandica has the genes necessary to synthesize the putative algal and bacterial lipids identified in our study, we utilized the KEGG PATHWAY database, which organizes current knowledge on metabolic pathways and records gene presence/absence data across species. KEGG PATHWAY suggests that A. queenslandica is missing genes necessary for the biosynthesis of saturated fatty acids (Figure 1A), sterols (Figure 1B), and unsaturated fatty acids (Figure 1C). However, the KEGG database makes its presence/absence determinations based on gene homology assignments against model organisms and does not necessarily account for nonhomologous genes with similar enzymatic functions, or for paralog/ ortholog nuances that arise during gene duplication events. For example, we previously demonstrated that A.queenslandica appears to contain the genes necessary to synthesize complex sterols (Gold, Grabenstatter, et al., 2016) but the KEGG PATHWAY map fails to identify many of these genes because it uses distantly related plant genes as models (Figure 1B). We therefore looked for each putative missing enzyme in the $A$. queenslandica genome to verify its presence or absence.
Regarding saturated fatty acids (Figure 1A), we found no evidence for the required fatty acid synthase-type enzyme in A. queenslandica, either the animal-homolog (FASN) or the equivalent enzymes in fungi (Fas1/Fas2) or bacteria (fas). A. queenslandica also appears to lack the BCKA decarboxylase enzyme (EC 4.1.1.72) necessary to synthesize the branched-chain fatty acids we recovered that are considered bacterial proxies (Kaneda, 1991; O'Reilly et al., 2016). However, A. queenslandica does contain some enzymes necessary for downstream fatty acid elongation, including 3-oxoacyl-[acyl-carrier-protein] synthase (FabF), 3-oxoacyl-[acyl-carrier protein] reductase (FabG), and enoyl-[acyl-carrier protein] reductase (Fabl). BLASTp searches against the NCBI protein database (accessed 06/27/2017) provide insight into the evolutionary history of these proteins. The Amphimedon FabF (NCBI accession: XP_003384093.1) has high sequence similarity with FabF-like proteins in other animals, while Fabl and FabG are most similar to bacterial proteins. The Amphimedon Fabl sequence (NCBI accession: XP_011407379.2) appears most similar to various Gammaproteobacteria, with the best hit coming from Thioalkalivibrio, followed by multiple taxa within the Methylococcales. Amphimedon FabG (NCBI accession: XP_019858191.1) is also most similar to Thioalkalivibrio, with the next best hits coming from various Acidobacteria and Alphaproteobacteria. These results suggest that FabF was laterally inherited, while Fabl and FabG were obtained through horizontal gene transfer with bacteria. However, we caution that these later proteins could also represent contamination in the A. queenslandica genome assembly; we initially found a putative FabZ-like protein in A. queenslandica, only to have it removed from NCBI after a recent update to the gene models.

Interpreting the genomic data for unsaturated fatty acids is more complicated (Figure 1C), as some of the missing enzymes are members of the large "fatty acid desaturase" and "elongation of very longchain fatty acids (elovl)" gene families. The A. queenslandica genome contains multiple copies of fatty acid desaturase and ELOVL-type proteins, so we constructed phylogenetic trees to determine their evolutionary relationships (Figure 2). A. queenslandica has putative homologs to ELOVL3, ELOVL6, and the ELOVL1/2/4/5/7 supergroup (Figure 2B). The divergence of ELOVL1,2,4,5, and 7 appear to represent gene duplications that occurred in bilaterian animals, following their divergence from the sponge lineage. It is therefore plausible that the single A. queenslandica protein (Uniprot ID: I1FDN3;
TABLE 2 Occurrence and average relative abundance ( $\mathrm{n}$ specimens $=4$ ) of sterols from Amphimedon queenslandica

\begin{tabular}{|c|c|c|}
\hline Sterols & & $\begin{array}{l}\text { Average } \pm \text { range } \\
(n=4)\end{array}$ \\
\hline cholest-5,22-dien-3 $\beta$-ol & $\operatorname{cis} 22-\mathrm{C}_{26} \Delta^{5,22}$ & $3.9 \pm 1.1$ \\
\hline cholest-5-en-3 $\beta$-ol (cholesterol) & $C_{27} \Delta^{5}$ & $36.0 \pm 8.4$ \\
\hline 24-methylcholest-5,22-dien-3 $\beta$-ol (brassicasterol) & $C_{28} \Delta^{5,22}$ & $7.9 \pm 2.2$ \\
\hline 24-methylcholest-5,24(28)-dien-3 $\beta$-ol & $C_{28} \Delta^{5,24(28)}$ & $11.4 \pm 1.7$ \\
\hline 24-methylcholest-5-en-3 $\beta$-ol (campesterol) & $C_{28} \Delta^{5}$ & $4.9 \pm 1.0$ \\
\hline 24-ethylcholest-5,22-dien-3 $\beta$-ol (stigmasterol) & $C_{29} \Delta^{5,22}$ & $4.3 \pm 1.0$ \\
\hline 24-ethylcholest-5-en-3 $\beta$-ol ( $\beta$-sitosterol) & $C_{29} \Delta^{5}$ & $26.3 \pm 2.7$ \\
\hline 24-ethylcholesta-5,24(28)-dien-3 $\beta$-ol (fucosterol) & $C_{29} \Delta^{5,24(28)}$ & $4.0 \pm 1.5$ \\
\hline Unknown $\mathrm{C}_{29}$ sterols $(n=3)$ & & $1.3 \pm 0.2$ \\
\hline
\end{tabular}


(a) FATTY ACID BIOSYNTHESIS
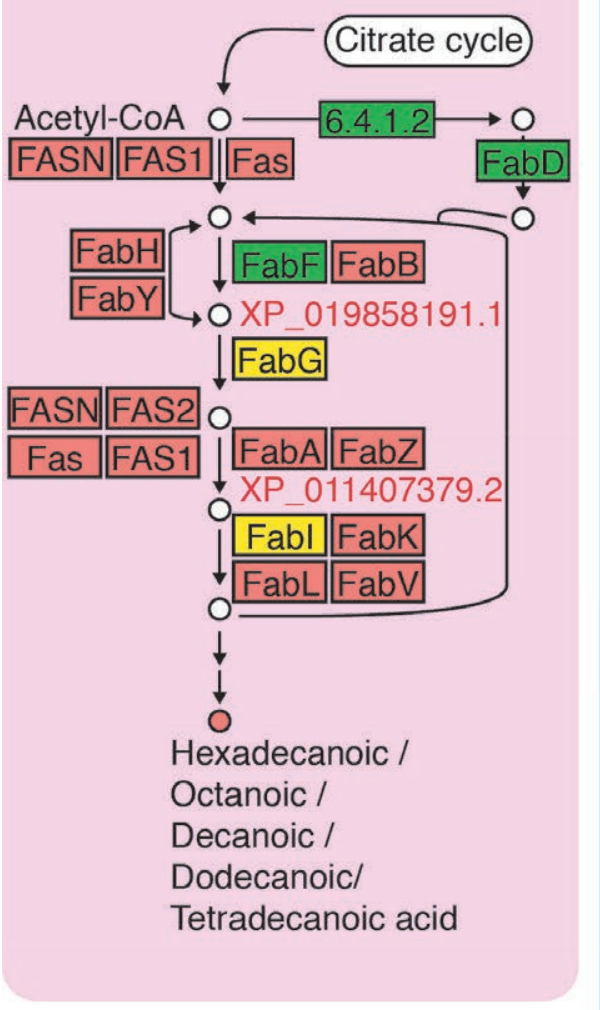

(b)
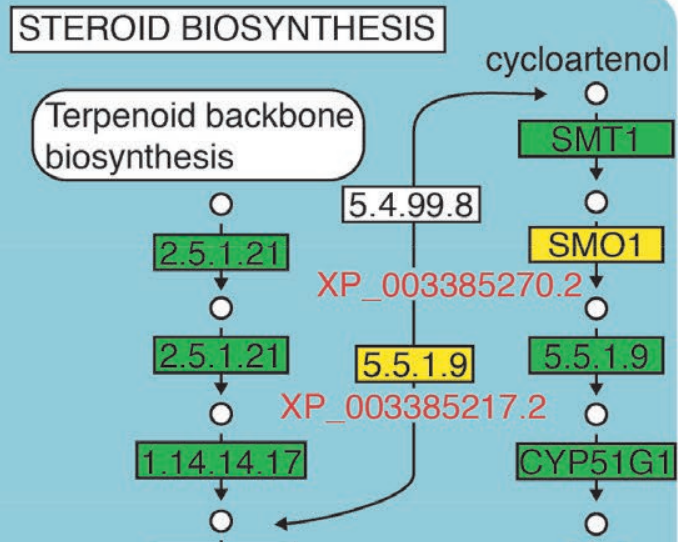

5.4.99.7

lanosterol

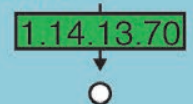

XP_003383101.2
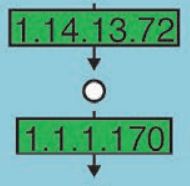

O cycloartenol

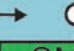

? MiT1 ○

SMO1

7.20

CYP51G1

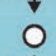

FK
XP_003383101.2

O

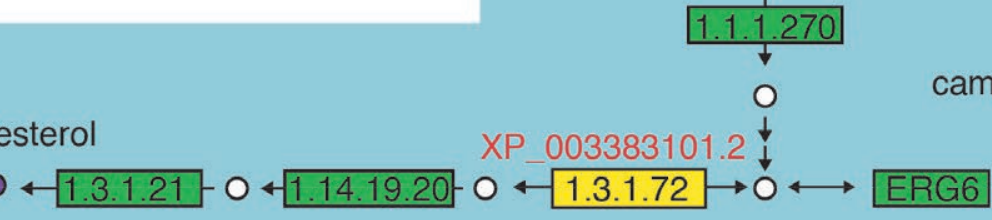

cholesterol

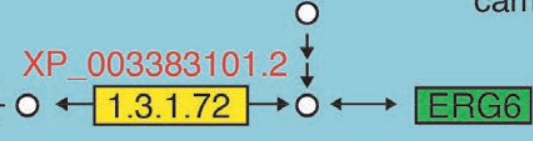

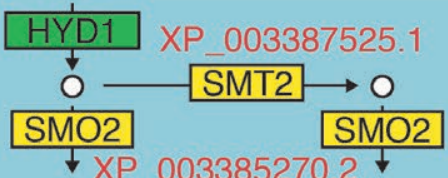

${ }_{0}^{\downarrow} X P \_003385270.2 \downarrow$

Enzyme present, recognized in KEGG

Enzyme present, not recognized in KEGG

Enzyme absent

Presence unclear

Molecule

Molecule of Interest

(c) BIOSYNTHESIS OF UNSATURATED FATTY ACIDS

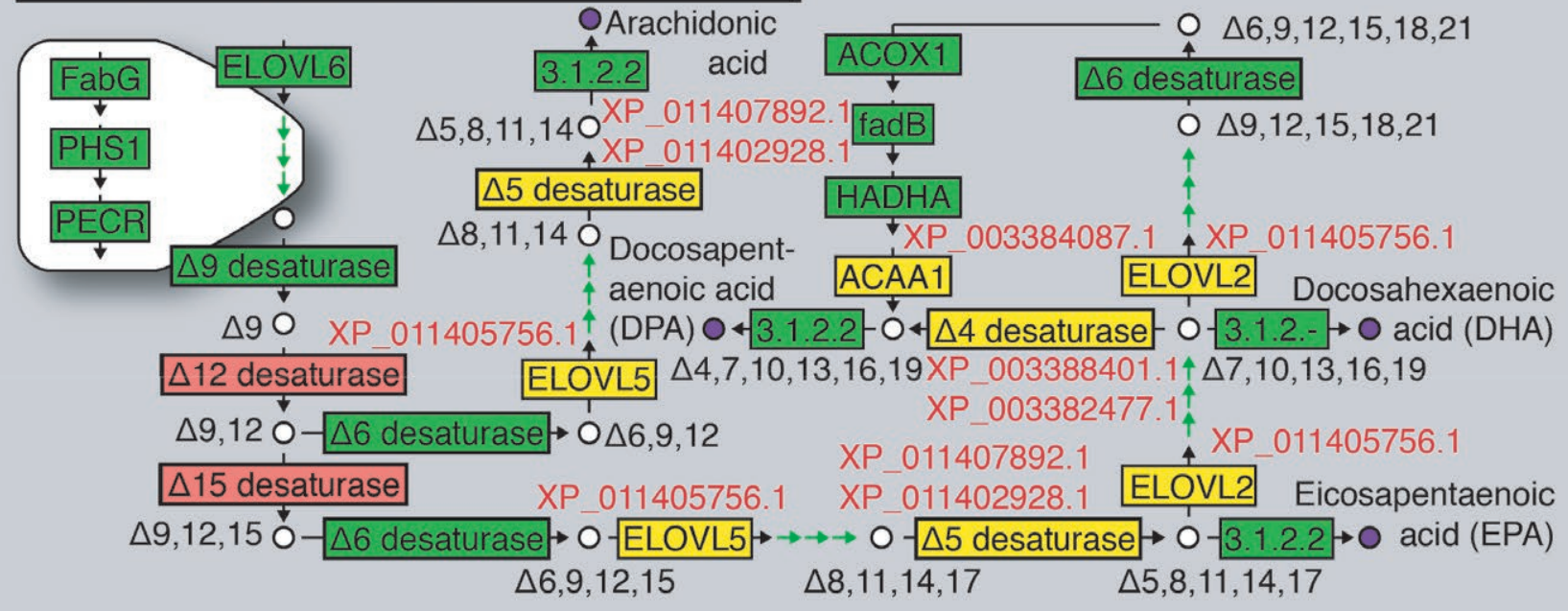

FIGURE 1 The presence and absence of Amphimedon queenslandica proteins involved in canonical lipid biosynthesis (based on the KEGG PATHWAY database). In cases where we recovered a putative protein from the A. queenslandica genome that is reported as "absent" in KEGG PATHWAY (yellow boxes), the NCBI accession number for the protein(s) is provided in red text. Pathways in this figure are redrawn from the original KEGG pathways: (a) fatty acid biosynthesis (pathway entry aqu00061), (b) steroid biosynthesis (aqu00100), (c) biosynthesis of unsaturated fatty acids (aqu01040) [Colour figure can be viewed at wileyonlinelibrary.com] 
(a)

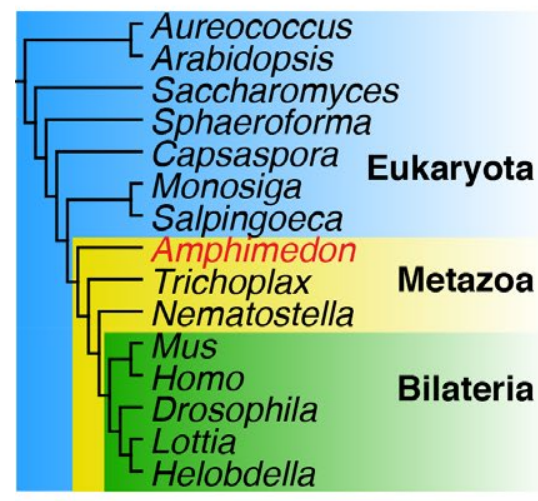

(b)

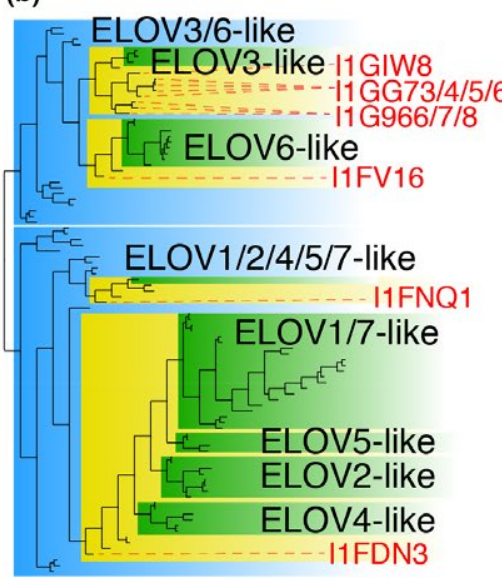

(c)

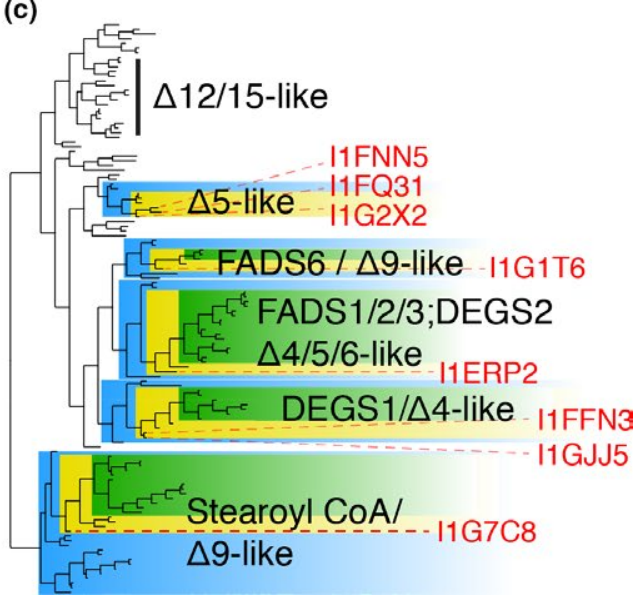

FIGURE 2 The evolution of Amphimedon queenslandica fatty acid desaturase and ELOVL proteins. (a) A phylogenetic tree showing the taxa used in this analysis. The colors distinguishing bilaterian animals (green) from non-bilaterian animals (yellow) and other eukaryotes (blue) are used in the protein trees to denote the taxonomy of relevant nodes. (b) A phylogenetic tree showing the evolutionary history of fatty acid desaturases, with A. queenslandica proteins noted with red UNIPROT IDs. (c) A phylogenetic tree showing the evolutionary history of ELOVL genes. Note that the trees in (b) and (c) represent maximum likelihood trees that have been optimized using species-tree guidance, as described in the Materials and Methods [Colour figure can be viewed at wileyonlinelibrary.com]

NCBI accession: XP_011405756.2) has functions related to both ELOVL2 and ELOVL5, which play separate roles in bilaterians during the biosynthesis of unsaturated fatty acids (Figure 1C). For the desaturase family (Figure $2 \mathrm{C}$ ), we recovered putative delta-4, delta-5, delta-6, and delta-9-like desaturases; however, the species lacks a member of the delta-12 fatty acid desaturase superfamily, which includes delta-12 and delta-15 desaturases. Ultimately, most of the genes reported as missing in the KEGG PATHWAY for unsaturated fatty acid biosynthesis do have one or more putative homologs in A. queenslandica, with the notable exception of those necessary for delta-12/15 desaturation.

\section{4 | DISCUSSION}

Based on our re-vetting of the genome data, we predict that $A$. queenslandica cannot produce basic fatty acids de novo, but that it should be able to modify certain fatty acids into the more complex "demospongic" and "algal" lipids recovered in our analysis. A. queenslandica lacks the enzymes necessary to initiate fatty acid synthesis (i.e., catalyze acetyl-CoA/malonyl-CoA into palmitic acid; Figure $1 \mathrm{~A}$ ), or create the branched fatty acids typically considered indicative of bacteria. It also lacks candidate delta-12 and delta-15 desaturases, suggesting it cannot synthesize basic $\Delta^{9,12}$ or $\Delta^{9,15}$ unsaturated fatty acids de novo (Figure 1C). But if given palmitic acid or $\Delta^{9,12 / 15}$ unsaturated fatty acids, A. queenslandica appears to have the additional enzymes necessary to generate delta-9 and delta- 5 modifications typical of demospongic acids, as well as putative "algal" proxies such as arachidonic acid, EPA, DPA, and DHA.

Amphimedon queenslandica similarly appears to have all genes necessary to synthesize $\mathrm{C}_{28}$ and $\mathrm{C}_{29}$ sterols de novo. Our analysis cannot definitively say what portion of the sterols found in A. queenslandica is generated by the sponge de novo, and what portion is obtained through diet or association with eukaryotic symbionts. However, the strong uniformity of sterol ratios across four individuals supports the hypothesis that sterol concentrations are actively regulated. This study supports several arguments regarding sponge biomarker genetics proposed in Gold, Grabenstatter, et al. (2016). Firstly, the presence of proteins such as CYP51G1, SMO2, STE1, and DWF1/5 suggests that demosponges actively produce sterols in the cycloartenol pathway, which is largely absent in most animals and fungi. The enzymes used to produce lanosterol (5.4.99.7 in Figure 1C) and cycloartenol (5.4.99.8) are orthologs of the same protein (oxidosqualene cyclase or OSC) so it is unclear if the single enzyme in A. queenslandica can produce both products. The amino acid composition of OSC in A. queenslandica supports lanosterol synthesis, although the presence of the CPL1 enzyme (5.5.1.9) suggests that this species can convert cycloartenol into lanosterol (Gold, Grabenstatter, et al., 2016). Additionally, the identification of diverse and abundant $\mathrm{C}_{28}$ and $\mathrm{C}_{29}$ sterols in A. queenslandica supports the hypothesis that conventional side-chain modifications can be synthesized by demosponges through the use of a promiscuous 24-sterol c-methyltransferase (smt) gene (Gold, Grabenstatter, et al., 2016; Gold, O'Reilly, et al., 2016). As A. queenslandica lacks a second smt paralog, we previously predicted that it cannot produce $\mathrm{C}_{30}$ sterols such as the "sponge biomarker" 24-isopropylcholesterol (Gold, Grabenstatter, et al., 2016), and our prediction has been borne out in this analysis.

Some caution and circumspection are necessary in the interpretation of our results. Although A. queenslandica appears to have the genes necessary to synthesize $\mathrm{C}_{28} / \mathrm{C}_{29}$ sterols and unsaturated fatty acids, this does not necessarily mean that the species actually produces these compounds in abundance. Fatty acid biosynthesis in A. queenslandica likely involves a combination of biosynthesis and the accumulation of lipid precursors taken up through diet 
and/or association with microbial symbionts, as has been found in other sponges (Carballeira, Thompson, Ayanoglu, \& Djerassi, 1986; Djerassi \& Lam, 1991; Hahn et al., 1988; Hochmuth et al., 2010; Raederstorff, Shu, Thompson, \& Djerassi, 1987). The genetic data must also be interpreted with caution: While A. queenslandica has enzymes broadly similar in sequence identity to those necessary for sterol and fatty acid production, it is possible that these genes actually represent unidentified paralogs, or have unique functions unrelated to lipid biosynthesis. It is also possible that sponges have evolved unique genes to perform certain enzymatic steps in lipid biosynthesis, as has been found in other eukaryotes (Desmond \& Gribaldo, 2009). Ultimately, the ability of A. queenslandica to synthesize the lipids identified in this study should be followed up with radiolabeling experiments, and additional demosponges should be analyzed in this manner to determine the enzymatic capabilities of demosponges more broadly.

Despite these uncertainties, this study supports the idea that many "algal" biomarkers in the geological record contain contributions from animal sources, although primary producer inputs are expected to dominate. Our results add to a growing body of evidence suggesting that marine invertebrates are an important and understudied contributor to the marine polyunsaturated fatty acid pool (Monroig, Tocher, \& Navarro, 2013). The strong correlation between certain demosponges lineages and unusual sterols (Love et al., 2009) or fatty acids (Schreiber, Wörheide, \& Thiel, 2006) suggests that these animals play a critical role in the biosynthesis of such compounds, even if they require precursor input from their diet or the holobiont. It is important to note that the presence of conventional $\mathrm{C}_{28} / \mathrm{C}_{29}$ steranes in the geologic record will never provide sufficient evidence for sponges; such inputs can only be recognized through unconventional steroid detection, with appropriate self-consistency checks using abundance ratios and demonstration of syngeneity (Love \& Summons, 2015). However, our results also suggest that the contribution of sponges to the conventional steroid pool cannot be discounted either.

Ultimately, the implications of this study for understanding sponge contributions to the geochemical record depends on the biomarker class. Polyunsaturated fatty acids rapidly oxidize in the environment, and finding these in environmental samples almost certainly indicates biomass or very fresh planktonic detritus. In other words, these diagnostic fatty acids have little use as biomarkers beyond modern settings. By contrast, $\mathrm{C}_{28} /{ }_{29}$ steranes (the diagenetic alteration products of precursor sterols) in rocks are overwhelmingly algal, as microalgae together with photosynthetic prokaryotes (which do not produce sterols) are the major contributors to modern marine organic matter. However, community ecology and structure have changed significantly over deep time, and sponges might have provided a significant contribution to the environmental lipid signal following mass extinction events (Botting et al., 2017; Ritterbush, Rosas, Corsetti, Bottjer, \& West, 2015), or in Neoproterozoic/early Phanerozoic rocks prior to the evolution of some algal lineages. In these scenarios, the possible contribution of sponges to the geochemical record should be considered.

\section{ACKNOWLEDGMENTS}

We gratefully acknowledge funding from the NASA Astrobiology Institute (NNA13AA90A) Foundations of Complex Life, Evolution, Preservation, and Detection on Earth and Beyond. DAG acknowledges support from an Agouron Institute Geobiology Fellowship, and SSOR acknowledges support from the Marie Skłodowska-Curie Actions Programme and the Irish Research Council (ELEVATE Postdoctoral Fellowship ELEVATEPD/2014/47).

\section{CONFLICT OF INTEREST}

The authors declare no conflict of interests.

\section{REFERENCES}

Botting, J. P., Muir, L. A., Zhang, Y., Ma, X., Ma, J., Wang, L., ... Fang, X. (2017). Flourishing sponge-based ecosystems after the End-Ordovician mass extinction. Current Biology, 27, 556-562.

Carballeira, N. M., \& Alicea, J. (2001). The first naturally occurring $\alpha$-methoxylated branched-chain fatty acids from the phospholipids of Amphimedon complanata. Lipids, 36, 83-87.

Carballeira, N. M., Negrón, V., \& Reyes, E. D. (1992). Novel Monounsaturated Fatty Acids from the Sponges Amphimedon compressa and Mycale laevis. Journal of Natural Products, 55, 333-339.

Carballeira, N. M., \& Shalabi, F. (1994). Unusual lipids in the Caribbean sponges Amphimedon viridis and Desmapsamma anchorata. Journal of Natural Products, 57, 1152-1159.

Carballeira, N., Thompson, J. E., Ayanoglu, E., \& Djerassi, C. (1986). Biosynthetic studies of marine lipids. 5. The biosynthesis of longchain branched fatty acids in marine sponges. The Journal of Organic Chemistry, 51, 2751-2756.

Chen, K., Durand, D., \& Farach-Colton, M. (2000). NOTUNG: a program for dating gene duplications and optimizing gene family trees. Journal of Computational Biology, 7, 429-447.

Desmond, E., \& Gribaldo, S. (2009). Phylogenomics of sterol synthesis: insights into the origin, evolution, and diversity of a key eukaryotic feature. Genome Biology and Evolution, 1, 364-381.

Djerassi, C., \& Lam, W. K. (1991). Phospholipid studies of marine organisms. Part 25. Sponge phospholipids.. Accounts of Chemical Research, 24, 69-75.

Eddy, S. R. (2008). A probabilistic model of local sequence alignment that simplifies statistical significance estimation. PLOS Computational Biology, 4, e1000069.

Edgar, R. C. (2004). MUSCLE: multiple sequence alignment with high accuracy and high throughput. Nucleic Acids Research, 32, 1792-1797.

Garson, M. J., Zimmermann, M. P., Battershill, C. N., Holden, J. L., \& Murphy, P. T. (1994). The distribution of brominated long-chain fatty acids in sponge and symbiont cell types from the tropical marine sponge Amphimedon terpenensis. Lipids, 29, 509-516.

Garson, M. J., Zimmermann, M. P., Hoberg, M., Larsen, R. M., Battershill, C. N., \& Murphy, P. T. (1993). Isolation of brominated long-chain fatty acids from the phospholipids of the tropical marine sponge Amphimedon terpenensis. Lipids, 28, 1011-1014.

Giner, J. L., Zhao, H., Boyer, G. L., Satchwell, M. F., \& Andersen, R. A. (2009). Sterol chemotaxonomy of marine pelagophyte algae. Chemistry \& Biodiversity, 6, 1111-1130.

Gold, D. A., Grabenstatter, J., de Mendoza, A., Riesgo, A., Ruiz-Trillo, I., \& Summons, R. E. (2016). Sterol and genomic analyses validate the sponge biomarker hypothesis. Proceedings of the National Academy of Sciences, 113, 2684-2689. 
Gold, D. A., O'Reilly, S. S., Luo, G., Briggs, D. E. G., \& Summons, R. E. (2016). Prospects for sterane preservation in sponge fossils from museum collections and the utility of sponge biomarkers for molecular clocks. Bulletin of the Peabody Museum of Natural History, 57, 181-189.

Hahn, S., Stoilov, I. L., Ha, T. B. T., Raederstorff, D., Doss, G. A., Li, H. T., \& Djerassi, C. (1988). Biosynthetic studies of marine lipids. 17. The course of chain elongation and desaturation in long-chain fatty acids of marine sponges. Journal of the American Chemical Society, 110, 8117-8124.

Hochmuth, T., Niederkrüger, H., Gernert, C., Siegl, A., Taudien, S., Platzer, M., ... Piel, J. (2010). Linking chemical and microbial diversity in marine sponges: possible role for Poribacteria as producers of Methylbranched fatty acids. ChemBioChem, 11, 2572-2578.

Hofheinz, W., \& Oesterhelt, G. (1979). 24-Isopropylcholesterol and 22-De hydro-24-isopropylcholesterol, novel sterols from a sponge. Helvetica Chimica Acta, 62, 1307-1309.

Kaneda, T. (1991). Iso- and anteiso-fatty acids in bacteria: biosynthesis, function, and taxonomic significance. Microbiology and Molecular Biology Reviews, 55, 288-302.

Kanehisa, M., \& Goto, S. (2000). KEGG: kyoto encyclopedia of genes and genomes. Nucleic Acids Research, 28, 27-30.

Kornprobst, J.-M., \& Barnathan, G. (2010). Demospongic acids revisited. Marine Drugs, 8, 2569-2577.

Lawson, M. P., Stoilov, I. L., Thompson, J. E., \& Djerassi, C. (1988). Cell membrane localization of sterols with conventional and unusual side chains in two marine demonsponges. Lipids, 1, 750-754.

Leys, S. P., Larroux, C., Gauthier, M., Adamska, M., Fahey, B., Richards, G. S., ... Degnan, B. M. (2008). Isolation of Amphimedon developmental material. Cold Spring Harbor Protocols, 2008(13), pdb.prot5095-pdb. prot5095.

Love, G. D., Grosjean, E., Stalvies, C., Fike, D. A., Grotzinger, J. P., Bradley, A. S., ... Summons, R. E. (2009). Fossil steroids record the appearance of Demospongiae during the Cryogenian period. Nature, 457, 718-721.

Love, G. D., \& Summons, R. E. (2015). The molecular record of Cryogenian sponges-a response to Antcliffe (2013). Palaeontology, 58, 1131-1136.

Monroig, Ó., Tocher, R. D., \& Navarro, C. J. (2013). Biosynthesis of polyunsaturated fatty acids in marine invertebrates: recent advances in molecular mechanisms. Marine Drugs, 11, 3998-4018.

Nichols, P. D., Guckert, J. B., \& White, D. C. (1986). Determination of monosaturated fatty acid double-bond position and geometry for microbial monocultures and complex consortia by capillary GC-MS of their dimethyl disulphide adducts. Journal of Microbiological Methods, 5, 49-55.

O'Reilly, S. S., Pentlavalli, P., Flanagan, P. V., Allen, C. C. R., Monteys, X., Szpak, M. T., ... Kelleher, B. P. (2016). Abundance and diversity of sedimentary bacterial communities in a coastal productive setting in the Western Irish Sea. Continental Shelf Research, 113, 1-9.
Raederstorff, D., Shu, A. Y. L., Thompson, J. E., \& Djerassi, C. (1987). Biosynthetic studies of marine lipids. 11. Synthesis, biosynthesis, and absolute configuration of the internally branched demospongic acid, 22-methyl-5,9-octacosadienoic acid. The Journal of Organic Chemistry, 52, 2337-2346.

Redmond, N. E., Raleigh, J., van Soest, R. W. M., Kelly, M., Travers, S. A. A., Bradshaw, B., ... McCormack, G. P. (2011). Phylogenetic relationships of the Marine Haplosclerida (Phylum Porifera) employing Ribosomal (28S rRNA) and Mitochondrial (cox1, nad1) gene sequence data. PLoS ONE, 6, e24344.

Ritterbush, K. A., Rosas, S., Corsetti, F. A., Bottjer, D. J., \& West, A. J. (2015). Andean sponges reveal long-term benthic ecosystem shifts following the end-Triassic mass extinction. Palaeogeography, Palaeoclimatology, Palaeoecology, 420, 193-209.

Schreiber, A., Wörheide, G., \& Thiel, V. (2006). The fatty acids of calcareous sponges (Calcarea, Porifera). Chemistry and Physics of Lipids, 143, 29-37.

Srivastava, M., Simakov, O., Chapman, J., Fahey, B., Gauthier, M. E. A., Mitros, T., ... Rokhsar, D. S. (2010). The Amphimedon queenslandica genome and the evolution of animal complexity. Nature, 466, 720-726.

Stamatakis, A. (2006). RAxML-VI-HPC: maximum likelihood-based phylogenetic analyses with thousands of taxa and mixed models. Bioinformatics, 22, 2688-2690.

Thiel, V., Blumenberg, M., Hefter, J., Pape, T., Pomponi, S., Reed, J., ... Michaelis, W. (2002). A chemical view of the most ancient metazoa-biomarker chemotaxonomy of hexactinellid sponges. Naturwissenschaften, 89, 60-66.

Thiel, V., Jenisch, A., Wörheide, G., Löwenberg, A., Reitner, J., \& Michaelis, W. (1999). Mid-chain branched alkanoic acids from "living fossil" demosponges: a link to ancient sedimentary lipids? Organic Geochemistry., $30,1-4$.

Volkman, J. K., Barrett, S. M., Blackburn, S. I., Mansour, M. P., Sikes, E. L., \& Gelin, F. (1998). Microalgal biomarkers: a review of recent research developments. Organic Geochemistry, 29, 1163-1179.

Watson, J., Brennan, T., Degnan, B., Degnan, S., \& Krömer, J. O. (2014). Analysis of the biomass composition of the Demosponge Amphimedon queenslandica on Heron Island Reef, Australia. Marine Drugs, 12, 3733-3753.

Watson, J., Degnan, B., Degnan, S., \& Krömer, J. O. (2014). Determining the biomass composition of a sponge holobiont for flux analysis. In J. O. Krömer, L. K. Nielsen, \& L. M. Blank (Eds.), Methods in molecular biology, Vol. 1191 (pp. 107-125). New York, NY: Springer, New York.

How to cite this article: Gold DA, O'Reilly SS, Watson J, et al. Lipidomics of the sea sponge Amphimedon queenslandica and implication for biomarker geochemistry. Geobiology.

2017;15:836-843. https://doi.org/10.1111/gbi.12253 